\title{
Existence of positive solutions of singular fractional differential equations with infinite-point boundary conditions
}

"Correspondence:

zhouzf12@126.com School of Mathematical Sciences, Anhui University, Hefei, Anhui 230601, China

\begin{abstract}
Using height functions of the nonlinear term on some bounded sets and considering integrations of these height functions, we obtain the existence of positive solutions for a class of singular fractional differential equations with infinite-point boundary value conditions.
\end{abstract}

Keywords: existence; positive solutions; singular fractional differential equation; infinite-point boundary value conditions

\section{Introduction}

In this paper, we investigate the existence of positive solutions of the following singular fractional differential equations with infinite-point boundary value conditions:

$$
\left\{\begin{array}{l}
D_{0^{+}}^{\alpha} x(t)+q(t) f(t, x(t))=0, \quad t \in(0,1) \\
x(0)=x^{\prime}(0)=\cdots=x^{(n-2)}(0)=0 \\
D_{0^{+}}^{\beta} x(1)=\sum_{i=1}^{\infty} \alpha_{i} x\left(\xi_{i}\right)
\end{array}\right.
$$

where $\alpha>2, n-1<\alpha \leq n, \beta \in[1, \alpha-1]$ is a fixed number, $\alpha_{i} \geq 0(i=1,2, \ldots), 0<\xi_{1}<\xi_{2}<$ $\cdots<\xi_{i-1}<\xi_{i}<\cdots<1, \frac{\Gamma(\alpha)}{\Gamma(\alpha-\beta)}-\sum_{i=1}^{\infty} \alpha_{i} \xi_{i}^{\alpha-1}>0$, and $D_{0^{+}}^{\alpha}$ and $D_{0^{+}}^{\beta}$ are the standard RiemannLiouville derivatives. The nonlinear term $f$ may be singular with respect to both time and space variables.

A function $x \in C[0,1]$ satisfying (1.1) is called a positive solution of (1.1) if $x>0$ on $(0,1]$. Recently, fractional differential equations have gained considerable attention; for example, see [1-5] and the references therein. Papers [6-9] discussed the existence of positive solutions of fractional boundary value problems.

In [10], the authors investigated the existence of a positive solution for the following fractional boundary value problem in a Banach space $E$ :

$$
\left\{\begin{array}{l}
D_{0^{+}}^{\alpha} u(t)+q(t) f(t, u(t))=0, \quad 0<t<1, \\
u(0)=u^{\prime}(0)=\cdots=u^{(n-2)}(0)=0, \\
u(1)=\sum_{i=1}^{m} \alpha_{i} u\left(\xi_{i}\right) .
\end{array}\right.
$$

(c) The Author(s) 2017. This article is distributed under the terms of the Creative Commons Attribution 4.0 International License (http://creativecommons.org/licenses/by/4.0/), which permits unrestricted use, distribution, and reproduction in any medium, provided you give appropriate credit to the original author(s) and the source, provide a link to the Creative Commons license, and indicate if changes were made. 
In [11], the authors obtained the existence and multiplicity of positive solutions of the following infinite-point fractional differential equations:

$$
\left\{\begin{array}{l}
D_{0^{+}}^{\alpha} u(t)+q(t) f(t, u(t))=0, \quad 0<t<1, \\
u(0)=0, \quad u(1)=\sum_{i=1}^{\infty} \alpha_{i} u\left(\xi_{i}\right) .
\end{array}\right.
$$

In [12], the author obtained the existence and multiplicity of positive solutions of the following fractional differential equation with infinite-point boundary value conditions:

$$
\left\{\begin{array}{l}
D_{0^{+}}^{\alpha} u(t)+q(t) f(t, u(t))=0, \quad 0<t<1, \\
u(0)=u^{\prime}(0)=\cdots=u^{(n-2)}(0)=0, \\
u^{(i)}(1)=\sum_{j=1}^{\infty} \alpha_{j} u\left(\xi_{j}\right) .
\end{array}\right.
$$

Motivated by the works mentioned, in this paper, we consider the boundary value problem (1.1) with more general and more complex boundary conditions. The existence of positive solutions for (1.1) is obtained by means of cone expansion and the compression fixed point theorem.

In order to establish the results, we assume that the following conditions are satisfied:

$\left(A_{1}\right) f \in C((0,1) \times(0,+\infty),[0,+\infty))$.

$\left(A_{2}\right) q \in C((0,1),[0,+\infty))$, and $q$ does not vanish identically on any subinterval of $(0,1)$.

$\left(A_{3}\right)$ For any positive numbers $r_{1}<r_{2}$, there exists a continuous function $p_{r_{1}, r_{2}}:(0,1) \rightarrow$ $[0,+\infty)$ such that

$$
\int_{0}^{1} q(t) p_{r_{1}, r_{2}}(t) d t<+\infty
$$

and

$$
f(t, u) \leq p_{r_{1}, r_{2}}(t), \quad 0<t<1, t^{\alpha-1} r_{1} \leq u \leq r_{2} .
$$

The rest of this paper is organized as follows. In Section 2, we introduce some preliminaries and relevant lemmas. In Section 3, we apply the fixed-point theorem to study the existence of positive solutions for the boundary value problem (1.1). In Section 4, we give some examples to illustrate our main results.

\section{Preliminaries and relevant lemmas}

For the convenience of the readers, we first present some useful definitions and fundamental facts of fractional calculus theory. In order to avoid redundancy, for the definitions of the Riemann-Liouville fractional integral and fractional derivative, we refer the readers to [13].

Lemma 2.1 ([14] $)$ Let $\alpha>0$, and $u(t)$ be an integrable function. Then

$$
I_{0^{+}}^{\alpha} D_{0^{+}}^{\alpha} u(t)=u(t)+c_{1} t^{\alpha-1}+c_{2} t^{\alpha-2}+\cdots+c_{n} t^{\alpha-n}
$$

where $c_{i} \in \mathbb{R}(i=1,2, \ldots, n)$, and $n$ is the smallest integer greater than or equal to $\alpha$. 
Lemma 2.2 Given $y \in C[0,1]$, the unique solution of the problem

$$
\left\{\begin{array}{l}
D_{0^{+}}^{\alpha} x(t)+y(t)=0, \quad t \in(0,1), \\
x(0)=x^{\prime}(0)=\cdots=x^{(n-2)}(0)=0, \\
D_{0^{+}}^{\beta} x(1)=\sum_{i=1}^{\infty} \alpha_{i} x\left(\xi_{i}\right),
\end{array}\right.
$$

is given by

$$
x(t)=\int_{0}^{1} G(t, s) y(s) d s
$$

where

$$
G(t, s)= \begin{cases}\frac{1}{\Gamma(\alpha) l(0)}\left\{l(s) t^{\alpha-1}(1-s)^{\alpha-\beta-1}-l(0)(t-s)^{\alpha-1}\right\}, & 0 \leq s \leq t \leq 1 \\ \frac{1}{\Gamma(\alpha) l(0)}\left\{l(s) t^{\alpha-1}(1-s)^{\alpha-\beta-1}\right\}, & 0 \leq t \leq s \leq 1\end{cases}
$$

with $l(s)=\frac{1}{\Gamma(\alpha-\beta)}-\frac{1}{\Gamma(\alpha)} \sum_{s \leq \xi_{i}} \alpha_{i}\left(\frac{\xi_{i}-s}{1-s}\right)^{\alpha-1}(1-s)^{\beta}(0 \leq s \leq 1)$.

Proof By Lemma 2.1 we can see that

$$
x(t)=-I_{0^{+}}^{\alpha} y(t)+c_{1} t^{\alpha-1}+c_{2} t^{\alpha-2}+c_{3} t^{\alpha-3}+\cdots+c_{n} t^{\alpha-n},
$$

so, the solution of (2.1) is

$$
x(t)=-\frac{1}{\Gamma(\alpha)} \int_{0}^{t}(t-s)^{\alpha-1} y(s) d s+c_{1} t^{\alpha-1}+c_{2} t^{\alpha-2}+c_{3} t^{\alpha-3}+\cdots+c_{n} t^{\alpha-n} .
$$

Since $x(0)=x^{\prime}(0)=\cdots=x^{(n-2)}(0)=0$, we have $c_{2}=c_{3}=\cdots=c_{n}=0$.

In addition,

$$
\begin{aligned}
D_{0^{+}}^{\beta} x(t)= & -D_{0^{+}}^{\beta} I_{0^{+}}^{\alpha} y(t)+c_{1} D_{0^{+}}^{\beta}\left(t^{\alpha-1}\right) \\
= & -\frac{1}{\Gamma(\alpha-\beta)} \int_{0}^{t}(t-s)^{\alpha-\beta-1} y(s) d s+c_{1} \frac{\Gamma(\alpha)}{\Gamma(n+\alpha-\beta)} \frac{d^{n}}{d t^{n}}\left(t^{n+\alpha-\beta-1}\right) \\
= & -\frac{1}{\Gamma(\alpha-\beta)} \int_{0}^{t}(t-s)^{\alpha-\beta-1} y(s) d s \\
& +c_{1} \frac{\Gamma(\alpha)}{\Gamma(n+\alpha-\beta)}(\alpha-\beta+n-1)(\alpha-\beta+n-2) \cdots(\alpha-\beta) t^{\alpha-\beta-1},
\end{aligned}
$$

where $n=[\beta]+1$.

Combining this with the second boundary value condition of (2.1), we have

$$
\begin{aligned}
D_{0^{+}}^{\beta} x(1) & =-\frac{1}{\Gamma(\alpha-\beta)} \int_{0}^{1}(1-s)^{\alpha-\beta-1} y(s) d s+c_{1} \frac{\Gamma(\alpha)}{\Gamma(\alpha-\beta)} \\
& =-\frac{1}{\Gamma(\alpha)} \sum_{i=1}^{\infty} \alpha_{i} \int_{0}^{\xi_{i}}\left(\xi_{i}-s\right)^{\alpha-1} y(s) d s+c_{1} \sum_{i=1}^{\infty} \alpha_{i} \xi_{i}^{\alpha-1} .
\end{aligned}
$$


This yields

$$
c_{1}=\frac{\frac{1}{\Gamma(\alpha-\beta)} \int_{0}^{1}(1-s)^{\alpha-\beta-1} y(s) d s-\frac{1}{\Gamma(\alpha)} \sum_{i=1}^{\infty} \alpha_{i} \int_{0}^{\xi_{i}}\left(\xi_{i}-s\right)^{\alpha-1} y(s) d s}{\frac{\Gamma(\alpha)}{\Gamma(\alpha-\beta)}-\sum_{i=1}^{\infty} \alpha_{i} \xi_{i}^{\alpha-1}} .
$$

Therefore, the solution of (2.1) is

$$
\begin{aligned}
x(t)= & -\frac{1}{\Gamma(\alpha)} \int_{0}^{t}(t-s)^{\alpha-1} y(s) d s+\frac{\frac{1}{\Gamma(\alpha-\beta)} \int_{0}^{1}(1-s)^{\alpha-\beta-1} t^{\alpha-1} y(s) d s}{\frac{\Gamma(\alpha)}{\Gamma(\alpha-\beta)}-\sum_{i=1}^{\infty} \alpha_{i} \xi_{i}^{\alpha-1}} \\
& -\frac{\frac{1}{\Gamma(\alpha)} \int_{0}^{1} \sum_{s \leq \xi_{i}} \alpha_{i}\left(\xi_{i}-s\right)^{\alpha-1} t^{\alpha-1} y(s) d s}{\frac{\Gamma(\alpha)}{\Gamma(\alpha-\beta)}-\sum_{i=1}^{\infty} \alpha_{i} \xi_{i}^{\alpha-1}} \\
= & \int_{0}^{1} G(t, s) y(s) d s .
\end{aligned}
$$

The proof is completed.

It is easy to see that if $l(s)$ is continuous on $[0,1]$, then $G(t, s)$ is continuous on $[0,1] \times$ $[0,1]$.

Lemma 2.3 The function $l(s)$ is positive and nondecreasing on $[0,1]$.

Proof By the definition of $l(s)$ it follows that

$$
l(0)=\frac{1}{\Gamma(\alpha-\beta)}-\frac{1}{\Gamma(\alpha)} \sum_{i=1}^{\infty} \alpha_{i} \xi_{i}^{\alpha-1}>0 .
$$

By computation we have that

(1) when $0 \leq s<\lim _{i \rightarrow \infty} \xi_{i}$,

$$
\begin{aligned}
l^{\prime}(s) & =\frac{1}{\Gamma(\alpha)}\left[\sum_{s \leq \xi_{i}} \alpha_{i}(\alpha-1)\left(\frac{\xi_{i}-s}{1-s}\right)^{\alpha-2}(1-s)^{\beta-2}\left(1-\xi_{i}\right)+\sum_{s \leq \xi_{i}} \alpha_{i} \beta\left(\frac{\xi_{i}-s}{1-s}\right)^{\alpha-1}(1-s)^{\beta-1}\right] \\
& >0, \quad \text { and }
\end{aligned}
$$

(2) when $\lim _{i \rightarrow \infty} \xi_{i} \leq s \leq 1$,

$$
l(s)=\frac{1}{\Gamma(\alpha-\beta)}, \quad l^{\prime}(s)=0 .
$$

So, $l$ is nondecreasing on $[0,1]$, and $l(s) \geq l(0)>0, s \in[0,1]$. The proof is completed.

Lemma 2.4 The function $G(t, s)$ defined in (2.2) admits the following properties:

(1) $G(t, s)>0, \frac{\partial}{\partial t} G(t, s)>0,0<t, s<1$;

(2) $\max _{t \in[0,1]} G(t, s)=G(1, s)=\frac{1}{\Gamma(\alpha) l(0)}\left[l(s)(1-s)^{\alpha-\beta-1}-l(0)(1-s)^{\alpha-1}\right], 0 \leq s \leq 1$;

(3) $G(t, s) \geq t^{\alpha-1} G(1, s), 0 \leq t, s \leq 1$.

Proof The proof is similar to that of Lemma 3 in [12].

The following lemma is the main tool in this paper. 
Lemma 2.5 ([16]) Let $E$ be a real Banach space, and $P \subset E$ be a cone. Assume that $\Omega_{1}$ and $\Omega_{2}$ are bounded open subsets of $E$ with $\theta \in \Omega_{1}, \overline{\Omega_{1}} \subset \Omega_{2}$, and $\left.T: P \cap \overline{\left(\Omega_{2}\right.} \backslash \Omega_{1}\right) \rightarrow P$ is a completely continuous operator such that either:

(1) $\|T u\| \leq\|u\|, u \in P \cap \partial \Omega_{1}$, and $\|T u\| \geq\|u\|, u \in P \cap \partial \Omega_{2}$, or

(2) $\|T u\| \geq\|u\|, u \in P \cap \partial \Omega_{1}$, and $\|T u\| \leq\|u\|, u \in P \cap \partial \Omega_{2}$.

Then $T$ has a fixed point in $P \cap\left(\overline{\Omega_{2}} \backslash \Omega_{1}\right)$.

Let $E=C[0,1]$, so that $E$ is a Banach space with norm $\|x\|=\max _{t \in[0,1]}|x(t)|$.

Set $P=\left\{x \in E \mid x(t) \geq t^{\alpha-1}\|x\|, t \in[0,1]\right\}$. Then $P$ is a cone in $E$.

Denote $\Omega(r)=\{x \in P:\|x\|<r\}$ and $\partial \Omega(r)=\{x \in P:\|x\|=r\}$ for $r>0$.

Define the operator $T: P \backslash\{\theta\} \rightarrow E$ by

$$
(T x)(t)=\int_{0}^{1} G(t, s) q(s) f(s, x(s)) d s, \quad t \in[0,1] .
$$

Lemma 2.6 Suppose that $\left(A_{1}\right)-\left(A_{3}\right)$ hold and $0<r_{1}<r_{2}$. Then $T: \overline{\Omega\left(r_{2}\right)} \backslash \Omega\left(r_{1}\right) \rightarrow P$ is completely continuous.

Proof For any $x \in \overline{\Omega\left(r_{2}\right)} \backslash \Omega\left(r_{1}\right)$, it follows from (2.3) and Lemma 2.4 that

$$
(T x)(t)=\int_{0}^{1} G(t, s) q(s) f(s, x(s)) d s \leq \int_{0}^{1} G(1, s) q(s) f(s, x(s)) d s, \quad t \in[0,1],
$$

and

$$
\begin{aligned}
(T x)(t) & =\int_{0}^{1} G(t, s) q(s) f(s, x(s)) d s \\
& \geq t^{\alpha-1} \int_{0}^{1} G(1, s) q(s) f(s, x(s)) d s, \quad t \in[0,1] .
\end{aligned}
$$

By (2.4) and (2.5) we know that

$$
(T x)(t) \geq t^{\alpha-1}\|T x\|, \quad t \in[0,1]
$$

which means that $T: \overline{\Omega\left(r_{2}\right)} \backslash \Omega\left(r_{1}\right) \rightarrow P$.

Noticing the continuity of $G(t, s)$ and $\left(A_{1}\right)-\left(A_{3}\right)$, it is easy to see that $T$ is continuous in $\overline{\Omega\left(r_{2}\right)} \backslash \Omega\left(r_{1}\right)$. Next, we show $T$ is compact. For any $x \in \overline{\Omega\left(r_{2}\right)} \backslash \Omega\left(r_{1}\right)$, we have

$$
|T x(t)|=\left|\int_{0}^{1} G(t, s) q(s) f(s, x(s)) d s\right| \leq \int_{0}^{1} G(1, s) q(s) p_{r_{1}, r_{2}}(s) d s
$$

for $t \in[0,1]$, which implies that $T\left(\overline{\Omega\left(r_{2}\right)} \backslash \Omega\left(r_{1}\right)\right)$ is uniformly bounded.

Because $G(t, s)$ is continuous on $[0,1] \times[0,1], G(t, s)$ is uniformly continuous on $[0,1] \times$ $[0,1]$. Thus, for any $\epsilon>0$, there exists $\delta>0$ such that

$$
\left|G\left(t_{1}, s\right)-G\left(t_{2}, s\right)\right|<\epsilon
$$


if $\left|t_{1}-t_{2}\right|<\delta$ and $\left(t_{1}, s\right),\left(t_{2}, s\right) \in[0,1] \times[0,1]$. Then, for any $x \in \overline{\Omega\left(r_{2}\right)} \backslash \Omega\left(r_{1}\right)$ and $t_{1}, t_{2} \in[0,1]$ such that $\left|t_{1}-t_{2}\right|<\delta$, we have

$$
\begin{aligned}
\left|T x\left(t_{1}\right)-T x\left(t_{2}\right)\right| & =\left|\int_{0}^{1}\left(G\left(t_{1}, s\right)-G\left(t_{2}, s\right)\right) q(s) f(s, x(s)) d s\right| \\
& \leq \int_{0}^{1}\left|G\left(t_{1}, s\right)-G\left(t_{2}, s\right)\right| q(s) p_{r_{1}, r_{2}}(s) d s \\
& \leq \epsilon \int_{0}^{1} q(s) p_{r_{1}, r_{2}}(s) d s .
\end{aligned}
$$

We can see that the functions in $T\left(\overline{\Omega\left(r_{2}\right)} \backslash \Omega\left(r_{1}\right)\right)$ are equicontinuous. So, $T\left(\overline{\Omega\left(r_{2}\right)} \backslash \Omega\left(r_{1}\right)\right)$ is relatively compact in $C[0,1]$. Thereby, $T$ is compact in $\overline{\Omega\left(r_{2}\right)} \backslash \Omega\left(r_{1}\right)$, and thus $T$ : $\overline{\Omega\left(r_{2}\right)} \backslash \Omega\left(r_{1}\right) \rightarrow P$ is completely continuous.

\section{Main result}

We introduce the following height functions to control the growth of the nonlinear term $f(t, x)$. Let

$$
\begin{array}{ll}
\varphi(t, r)=\max \left\{f(t, x): t^{\alpha-1} r \leq x \leq r\right\} & (0<t<, r>0), \\
\psi(t, r)=\min \left\{f(t, x): t^{\alpha-1} r \leq x \leq r\right\} & (0<t<1, r>0) .
\end{array}
$$

Theorem 3.1 Suppose that $\left(A_{1}\right)-\left(A_{3}\right)$ hold and there exist two positive numbers $a<b$ such that one of the following conditions is satisfied:

$\left(B_{1}\right) a \leq \int_{0}^{1} G(1, s) q(s) \psi(s, a) d s<+\infty$ and $\int_{0}^{1} G(1, s) q(s) \varphi(s, b) d s \leq b$;

$\left(B_{2}\right) \int_{0}^{1} G(1, s) q(s) \varphi(s, a) d s \leq a$ and $b \leq \int_{0}^{1} G(1, s) q(s) \psi(s, b) d s<+\infty$.

Then the boundary value problem (1.1) has at least one strictly increasing positive solution $x^{*} \in P$ such that $a \leq\left\|x^{*}\right\| \leq b$.

Proof Without loss of generality, we only prove $\left(B_{1}\right)$.

If $x \in \partial \Omega(a)$, then $\|x\|=a$ and $t^{\alpha-1} a \leq x(t) \leq a, 0 \leq t \leq 1$. By the definition of $\psi(t, a)$ we know that

$$
f(t, x(t)) \geq \psi(t, a), \quad 0<t<1
$$

By (3.1) and Lemma 2.4 we have that

$$
\begin{aligned}
\|T x\| & =\max _{t \in[0,1]} \int_{0}^{1} G(t, s) q(s) f(s, x(s)) d s \\
& \geq \max _{t \in[0,1]} t^{\alpha-1} \int_{0}^{1} G(1, s) q(s) f(s, x(s)) d s \\
& =\int_{0}^{1} G(1, s) q(s) f(s, x(s)) d s \\
& \geq \int_{0}^{1} G(1, s) q(s) \psi(s, a) d s \geq a=\|x\| .
\end{aligned}
$$


If $x \in \partial \Omega(b)$, then $\|x\|=b$ and $t^{\alpha-1} b \leq x(t) \leq b, 0 \leq t \leq 1$. By the definition of $\varphi(t, b)$ we get that

$$
f(t, x(t)) \leq \varphi(t, b), \quad 0<t<1
$$

By (3.3) and Lemma 2.4 we have that

$$
\begin{aligned}
\|T x\| & =\max _{t \in[0,1]} \int_{0}^{1} G(t, s) q(s) f(s, x(s)) d s \leq \int_{0}^{1} G(1, s) q(s) f(s, x(s)) d s \\
& \leq \int_{0}^{1} G(1, s) q(s) \varphi(s, b) d s \leq b=\|x\| .
\end{aligned}
$$

By Lemma 2.5, $T$ has a fixed point $x^{*} \in \overline{\Omega(b)} \backslash \Omega(a)$. From Section 2 we know that $x^{*}$ is a solution of (1.1) and $a \leq\left\|x^{*}\right\| \leq b$. Because $x^{*}(t) \geq t^{\alpha-1}\left\|x^{*}\right\| \geq a t^{\alpha-1}>0,0<t \leq 1$, we get that $x^{*}$ is a positive solution for (1.1).

From Lemma 2.4 we have that

$$
\left(x^{*}\right)^{\prime}(t)=\left(T x^{*}\right)^{\prime}(t)=\int_{0}^{1} \frac{\partial}{\partial t} G(t, s) q(s) f\left(s, x^{*}(s)\right) d s>0,
$$

which shows that $x^{*}$ is a strictly increasing positive solution. The proof is completed.

\section{Examples}

Example 4.1 Consider the boundary value problem

$$
\left\{\begin{array}{l}
D_{0^{+}}^{\frac{7}{2}} x(t)+\frac{1}{4 \sqrt{1-t}}\left(x^{5}+\frac{1}{2 \sqrt[3]{x}}\right)=0, \quad t \in(0,1) \\
x(0)=x^{\prime}(0)=x^{(2)}(0)=0 \\
D_{0^{+}}^{\frac{3}{2}} x(1)=\sum_{i=1}^{\infty} \frac{2}{i^{2}} x\left(1-\frac{1}{i+1}\right)
\end{array}\right.
$$

Let $\alpha=\frac{7}{2}, \beta=\frac{3}{2}, f(t, x)=x^{5}+\frac{1}{2 \sqrt[3]{x}}, q(t)=\frac{1}{4 \sqrt{1-t}}, \alpha_{i}=\frac{2}{i^{2}}, \xi_{i}=1-\frac{1}{i+1}$. Obviously, $f \in$ $C((0,1) \times(0,+\infty),[0,+\infty))$ and $q \in C((0,1),[0,+\infty))$. It is not difficult to calculate that $\sum_{i=1}^{\infty} \alpha_{i} \xi_{i}^{\alpha-1} \approx 1.643, \Gamma(\alpha)=\Gamma\left(\frac{7}{2}\right) \approx 3.3234, \Gamma(\alpha-\beta)=\Gamma(2)=1, l(0) \approx 0.8169$. For any positive numbers $r_{1}<r_{2}$, we can see that $\left(A_{1}\right)-\left(A_{3}\right)$ hold for $p_{r_{1}, r_{2}}(t)=r_{2}^{5}+\frac{1}{2} t^{-\frac{5}{6}} r_{1}^{-\frac{1}{3}}$.

The height functions $\varphi(t, r)$ and $\psi(t, r)$ satisfy the following inequalities:

$$
\begin{aligned}
& \varphi(t, r)=\max \left\{x^{5}+\frac{1}{2 \sqrt[3]{x}}: t^{\frac{5}{2}} r \leq x \leq r\right\} \leq r^{5}+\frac{1}{2} t^{-\frac{5}{6}} r^{-\frac{1}{3}}, \\
& \psi(t, r)=\min \left\{x^{5}+\frac{1}{2 \sqrt[3]{x}}: t^{\frac{5}{2}} r \leq x \leq r\right\} \geq t^{\frac{25}{2}} r^{5}+\frac{1}{2} r^{-\frac{1}{3}}
\end{aligned}
$$

It follows that

$$
\begin{aligned}
\int_{0}^{1} G(1, s) q(s) \varphi(s, 1) d s & \leq \frac{1}{\Gamma\left(\frac{7}{2}\right) l(0)} \int_{0}^{1}\left[l(s)(1-s)^{\alpha-\beta-1}\right] \frac{1}{4 \sqrt{1-s}}\left(1+\frac{1}{2} s^{-\frac{5}{6}}\right) d s \\
& \leq \frac{1}{\Gamma\left(\frac{7}{2}\right) l(0)} \int_{0}^{1} \Gamma(2)(1-s) \frac{1}{4 \sqrt{1-s}}\left(1+\frac{1}{2} s^{-\frac{5}{6}}\right) d s<1
\end{aligned}
$$


and

$$
\begin{aligned}
& \int_{0}^{1} G(1, s) q(s) \psi\left(s, \frac{1}{100}\right) d s \\
& \geq \frac{1}{\Gamma\left(\frac{7}{2}\right) l(0)} \int_{0}^{1}\left[l(s)(1-s)^{\alpha-\beta-1}-l(0)(1-s)^{\alpha-1}\right] \\
& \quad \times \frac{1}{4 \sqrt{1-s}}\left[s^{\frac{25}{2}}\left(\frac{1}{100}\right)^{5}+\frac{1}{2}\left(\frac{1}{100}\right)^{-\frac{1}{3}}\right] d s \\
& \geq \frac{1}{4 \Gamma\left(\frac{7}{2}\right) l(0)} \int_{0}^{1}\left[l(0)(1-s)-l(0)(1-s)^{\frac{5}{2}}\right](1-s)^{-\frac{1}{2}}\left[s^{\frac{25}{2}}\left(\frac{1}{100}\right)^{5}+\frac{1}{2}\left(\frac{1}{100}\right)^{-\frac{1}{3}}\right] d s \\
& >\frac{1}{100} .
\end{aligned}
$$

According to Theorem 3.1, we get that (4.1) has at least one strictly increasing positive solution $x^{*}$ and $\frac{1}{100} \leq\left\|x^{*}\right\| \leq 1$.

\section{Conclusions}

Infinite-point boundary value conditions are the classical boundary value conditions. The existence of positive solutions for singular fractional differential problem with infinitepoint boundary conditions is established by height functions of the nonlinear term on some bounded sets.

\section{Competing interests}

The authors declare that they have no competing interests.

\section{Authors' contributions}

The authors have equal contributions to each part of this paper. All authors read and approved the final manuscript.

\section{Acknowledgements}

This article was supported by the National Natural Science Foundation of China (Grant No. 11371027) and Anhui Provincial Natural Science Foundation (1608085MA12). The authors would like to thank the referees for their valuable suggestions and comments.

Received: 4 September 2016 Accepted: 18 November 2016 Published online: 09 January 2017

\section{References}

1. Agarwal, RP: Certain fractional $q$-integrals and q-derivatives. Math. Proc. Camb. Philos. Soc. 66, 365-370 (1969)

2. Al-Salam, WA: Some fractional $q$-integrals and q-derivatives. Proc. Edinb. Math. Soc. 15, 135-140 (1966)

3. Krasnoselskii, MA: Positive Solutions of Operator Equations. Noordhoff, Groningen (1964)

4. Zhou, Y, Peng, L: On the time-fractional Navier-Stokes equations. Comput. Math. Appl. (2016) in press. doi:10.1016/j.camwa.2016.03.026

5. Yao, Q: Local existence of multiple positive solutions to a singular cantilever beam equation. J. Math. Anal. Appl. 363, 138-154 (2010)

6. Zhou, Y, Vijayakumar, V, Murugesu, R: Controllability for fractional evolution inclusions without compactness. Evol. Equ. Control Theory 4, 507-524 (2015)

7. Zhao, Y, Sun, S, Han, Z, Li, Q: Positive solutions to boundary value problems of nonlinear fractional differential equations. Abstr. Appl. Anal. 2011, Article ID 390543 (2011)

8. Zhou, Y, Peng, L: Weak solution of the time-fractional Navier-Stokes equations and optimal control. Comput. Math. Appl. (2016) in press. doi:10.1016/j.camwa.2016.07.007

9. Ahmad, B, Nieto, JJ: Existence of solutions for anti-periodic boundary value problems involving fractional differential equations via Leray-Schauder degree theory. Topol. Methods Nonlinear Anal. 35, 295-304 (2010)

10. Wang, Y, Liu, L: Positive solutions for fractional $m$-point boundary value problem in Banach spaces. Acta Math. Sci. $32 \mathrm{~A}, 246-256(2012)$

11. Gao, H, Han, X: Existence of positive solutions for fractional differential equation with nonlocal boundary condition. Int. J. Differ. Equ. 2011, 10 (2011)

12. Zhang, $X$ : Positive solutions for a class of singular fractional differential equation with infinite-point boundary value conditions. Appl. Math. Lett. 39, 22-27 (2015)

13. Cui, Y: Uniqueness of solution for boundary value problems for fractional differential equations. Appl. Math. Lett. 51 48-54 (2016) 
14. Jiang, D, Yuan, C: The positive properties of the green function for Dirichlet-type boundary value problems of nonlinear fractional differential equations and its application. Nonlinear Anal. TMA 72, 710-719 (2010)

15. Zhang, $X$, Wang, L, Sun, Q: Existence of positive solutions for a class of nonlinear fractional differential equations with integral boundary conditions and a parameter. Appl. Math. Comput. 226, 708-718 (2014)

16. Zhang, $X, L i u, L$, Wiwatanapataphee, $B, W u, Y$ : The eigenvalue for a class of singular $p$-Laplacian fractional differential equations involving the Riemann-Stieltjes integral boundary condition. Appl. Math. Comput. 235, 412-422 (2014)

17. Zhang, $X, L i u, L, W u, Y$ : The uniqueness of positive solution for a fractional order model of turbulent flow in a porous medium. Appl. Math. Lett. 37, 26-33 (2014)

18. Wang, $L$, Zhang, $X$ : Positive solutions of $m$-point boundary value problems for a class of nonlinear fractional differential equations. J. Appl. Math. Comput., Int. J. 42, 387-399 (2013)

19. Zhou, Y: Fractional Evolution Equations and Inclusions: Analysis and Control. Academic Press, San Diego (2016)

20. Zhang, X: Positive solutions of singular higher-order fractional differential equations with nonlocal conditions. J. Appl. Math. Comput., Int. J. 49, 69-89 (2015)

21. Wang, Y, Liu, L, Wu, Y: Positive solutions for a nonlocal fractional differential equation. Nonlinear Anal. 74, 3599-3605 (2011)

\section{Submit your manuscript to a SpringerOpen ${ }^{\mathcal{O}}$ journal and benefit from:}

- Convenient online submission

- Rigorous peer review

Immediate publication on acceptance

Open access: articles freely available online

- High visibility within the field

- Retaining the copyright to your article 